\title{
NON-LINEAR EFFECTS IN THE EASY-PLANE ONE-DIMENSIONAL ANTIFERROMAGNET IN A STAGGERED FIELD
}

\author{
F.S.L. Cassino \\ Universidade Federal de Ouro Preto, Departamento de Física, 35400, Ouro Preto, MG, Brazil \\ and \\ A.S.T. Pires \\ UFMG, Departamento de Física, CP702, Belo Horizonte, 30161, MG, Brazil
}

(Received 4 January 1991 by P. Burlet)

\begin{abstract}
We present a theoretical analysis of soliton excitations in an easy-plane classical one-dimensional antiferromagnet in the presence of a staggered magnetic field transverse to the chain direction. The out-of-plane soliton solution is found to have an instability similar to the one found before by Magyari-Thomas-Kumar for the easy-plane ferromagnetic chain in an external magnetic field.
\end{abstract}

INVESTIGATIONS of nonlinear excitations in Heisenberg antiferromagnetic chain have in the later years received much attention. The properties of the classical one-dimensional anisotropic antiferromagnet in an external magnetic field have been treated extensively [1]. However, little attention has been paid to the case of a staggered magnetic field $[2,3]$. The study of this case is important not only for academic reasons, but also because it has some physical interest. For instance, below the critical temperature of an anisotropic quasi-one-dimensional antiferromagnet there is a long range magnetic order in the system of weakly interacting chains, such that the value of the staggered spontaneous magnetization does not equal to zero. One approach to study this model is to approximate the quasi-one-dimensional system by a single chain in the presence of the interchain staggered mean field [3].

In this paper we will be concerned with the onedimensional antiferromagnetic system with single-ion anisotropy $A\left(S^{z}\right)^{2}$ in external, ordinary and staggered, magnetic fields as described by the Hamiltonian,

$$
\begin{aligned}
H= & J \sum_{n} \mathbf{S}_{n} \mathbf{S}_{n+1}+A \sum_{n}\left(S_{n}^{z}\right)^{2}-g \mu_{B} H_{y} \sum_{n} S_{n}^{y} \\
& -g \mu_{B} H_{s} \sum_{n}(-1)^{n} S_{n}^{x} .
\end{aligned}
$$

Here $J>0$ and $A>0$, an easy-plane anisotropy. The case $A<0$, easy-axis (Ising-like) anisotropy together with a staggered field in the $z$-direction has been studied by Mikeska [2]. The classical ground state configuration of equation (1) is Ising-like, $\mathbf{S}=( \pm S, 0,0)$. For small ordinary magnetic fields $H_{y}$ we expect that two neighbouring spins are almost anti-parallel to each other at low temperatures, so that it is convenient to use the parametrization introduced by Mikeska [2].

$$
\begin{aligned}
& \mathbf{S}_{n}=(-1)^{n} S\left\{\sin \left(\theta_{n}+(-1)^{n} v_{n}\right) \cos \left(\phi_{n}+(-1)^{n} \varphi_{n}\right),\right. \\
& \sin \left(\theta_{n}+(-1)^{n} v^{n}\right), \quad \sin \left(\phi_{n}+(-1)^{n} \varphi_{n}\right), \\
& \cos \left(\theta_{n}+(-1)^{n} v_{n}\right) .
\end{aligned}
$$

$\theta$ and $\phi$ are angles giving the sublattice magnetization, whereas $v$ and $\varphi$ describe the deviations from perfect anti-alignment, and can be assumed to be small at low temperatures. Substituting equation (2) into the Hamiltonian (1) we can keep only the terms up to the second order in the small quantities $v, \varphi$ and the spatial variations of $\theta$ and $\phi$. We obtain in the continuum approximation

$$
\begin{aligned}
H= & \frac{J S}{2} \int \mathrm{d} z\left\{\left(\frac{\partial \theta}{\partial z}\right)^{2}+4 v^{2}\right. \\
& +\sin ^{2} \theta\left[4 \varphi^{2}+\left(\frac{\partial \phi}{\partial z}\right)^{2}\right] \\
& +K\left[\cos ^{2} \theta+v^{2}\left(1-2 \cos ^{2} \theta\right)\right] \\
& -2 h_{y}(\varphi \sin \theta \cos \phi+v \cos \theta \sin \phi) \\
& \left.-2 h_{\mathrm{s}} \sin \theta \cos \phi\right\},
\end{aligned}
$$

where

$$
K=\frac{2 A}{J}, \quad h_{\alpha}=\frac{g \mu_{B} H_{\alpha}}{J S}, \quad(\alpha=y, s),
$$

and $z$ is the coordinate along the chain. 
The equations of motion can be obtained either directly by applying the continuum approximation to the equations of motion on the discrete lattice or from the Hamiltonian (3). We obtain

$\frac{1}{J S} \frac{\partial \theta}{\partial t}=4 \varphi \sin \theta-\left(h_{y}-h_{s} \varphi\right) \cos \phi$,

$$
\begin{aligned}
\frac{1}{J S} \frac{\partial \phi}{\partial t}= & -\frac{4 v}{\sin \theta}-K v \sin \theta \\
& +\left(h_{y}-h_{s} \varphi\right) \cot \theta \sin \phi-h_{s} v \frac{\cos \phi}{\sin ^{2} \theta},
\end{aligned}
$$

$$
\begin{aligned}
\frac{1}{J S} \frac{\partial v}{\partial t}= & -4 v \varphi \cos \theta-2 \cos \theta \frac{\partial \theta}{\partial z} \frac{\partial \phi}{\partial \theta} \\
& -\sin \theta \frac{\partial^{z} \phi}{\partial z^{2}}+\left(h_{s}+h_{y} \varphi\right) \sin \phi \\
\frac{1}{J S} \frac{\partial \varphi}{\partial t}= & 4 v^{2} \frac{\cos }{\sin ^{2} \theta}-\cos \theta\left[4 \varphi^{2}+\left(\frac{\partial \phi}{\partial z}\right)^{2}\right] \\
& +\frac{1}{\sin \theta} \frac{\partial^{2} \theta}{\partial z^{2}}+K \cos \theta \\
& +\left(h_{\mathrm{s}}-h_{y} \varphi\right) \cot \theta \cos \phi-h_{y} v \frac{\sin ^{2}}{\sin ^{2} \theta} .
\end{aligned}
$$
obtain

After eliminating the small angles $v$ and $\varphi$, we

$$
\begin{aligned}
& \frac{\partial^{2} \theta}{\partial z^{2}}-\frac{1}{C^{2}} \frac{\partial^{2} \theta}{\partial t^{2}}=\sin \theta \cos \theta\left[\left(\frac{\partial \phi}{\partial z}\right)^{2}\right. \\
& \left.-\frac{1}{C^{2}}\left(\frac{\partial \phi}{\partial t}\right)^{2}\right]-K \sin \theta \cos \theta \\
& -h_{s} \cos \theta \cos \phi-\frac{h_{s}^{2}}{4} \cot \theta \cos ^{2} \phi \\
& +\frac{h_{y}^{2}}{4} \sin \theta \cos \theta \sin ^{2} \phi-\frac{h_{y}}{C} \sin ^{2} \theta \sin \phi \frac{\partial \phi}{\partial t},
\end{aligned}
$$

$$
\begin{aligned}
& \frac{\partial^{2} \phi}{\partial z^{2}}-\frac{1}{C^{2}} \frac{\partial^{2} \phi}{\partial t^{2}}=-2 \cot \theta\left(\frac{\partial \theta}{\partial z} \frac{\partial \phi}{\partial z}\right. \\
& \left.-\frac{1}{C^{2}}-\frac{\partial \theta}{\partial t} \frac{\partial \phi}{\partial t}\right)+\frac{h_{s}}{4}\left(4+K \cos ^{2} \theta\right) \frac{\sin \phi}{\sin \theta} \\
& +h_{s}^{2} \cot \theta \sin \phi \cos \phi \\
& +\frac{h_{y}}{4} \sin \phi \cos \phi+\frac{h_{y}}{C} \sin \phi \frac{\partial \theta}{\partial t},
\end{aligned}
$$

$$
\begin{aligned}
E_{x y}^{0}= & 4 J S^{2} \sqrt{h_{s}}\left\{(1+\tilde{\lambda})^{1 / 2}+\frac{1}{2 \tilde{\lambda}^{1 / 2}}\right. \\
& \left.\times \ln \left[\frac{(1+\tilde{\lambda})^{1 / 2}+\tilde{\lambda}^{1 / 2}}{(1+\tilde{\lambda})^{1 / 2}-\tilde{\lambda}^{1 / 2}}\right]\right\}
\end{aligned}
$$

As we can see, when $\tilde{\lambda} \rightarrow 0$ we find $E_{x y}^{0}=8 J S^{2} \sqrt{h_{s}}$ the energy of the static sine-Gordon soliton [2].

Another solution to equations (8) and (9) is given by $\phi=0$ and $\theta(z, t)$ satisfying

$\frac{\partial^{2} \theta}{\partial z^{2}}-\frac{1}{C^{2}} \frac{\partial^{2}}{\partial t^{2}}=-K \sin \theta \cos \theta$

$$
-h_{s} \cos \theta-\frac{h_{s}^{2}}{4} \cot \theta
$$

Taking $\theta=\psi+\pi / 2$ and neglecting the last term on the right hand side of equation (13) (it is second order in the small quantity $h_{s}$ ) we obtain

$\frac{\partial^{2} \psi}{\partial z^{2}}-\frac{1}{C^{2}} \frac{\partial^{2} \Psi}{\partial t^{2}}=K \sin \psi \cos \psi+h_{\mathrm{s}} \sin \psi$,

the dynamical DSG equation. The $x z$ kink is then given by

$$
\begin{aligned}
\theta(z, t)= & \pm 2 \arctan \left\{(1+\lambda)^{1 / 2}\right. \\
& \left.\times \operatorname{cosech}\left[\gamma \sqrt{h_{s}}(1+\lambda)^{1 / 2}(z-u t)\right]\right\}+\frac{\pi}{2},
\end{aligned}
$$

where $\lambda=K / h_{s}, \gamma=\left(1-u^{2} / c^{2}\right)^{-1 / 2}$, and $u$ is the kink velocity. The static $x z$ kink energy is

$$
\begin{aligned}
E_{x z}^{0}= & 4 J S^{2} \sqrt{h_{s}}\left\{(1+\lambda)^{1 / 2}\right. \\
& \left.+\frac{1}{2 \lambda^{1 / 2}} \ln \left(\frac{(1+\lambda)^{1 / 2}+\lambda^{1 / 2}}{(1+\lambda)^{1 / 2}-\lambda^{1 / 2}}\right)\right\} .
\end{aligned}
$$


Depending on the ration $r=h_{y} / 2 K^{1 / 2}$ the $x y$ kink $(r<1$, easy $x y$ plane) or the $x z$ kink $(r>1$, easy $x z$ plane) has lower energy. For $r=1$ there is one soliton with rotational degeneracy.

Now we will investigate a time-dependent solution for equations (8) and (9) for slowly moving $x y$ kinks. We make the ansatz,

$\theta_{x y}(z, t)=\frac{\pi}{2}+\theta_{0}(s)+\mathrm{O}\left(u^{3}\right)$

$\phi_{x y}(z, t)=\phi_{0}(s)+\mathrm{O}\left(u^{2}\right)$,

where $s=z-u t$. Inserting equations (17) into equations (8) and (9) we find

$$
\begin{aligned}
\frac{\mathrm{d}^{2} \theta_{0}}{\mathrm{~d} s^{2}}= & -\theta_{0}\left(\frac{\mathrm{d} \phi_{0}}{\mathrm{~d} s}\right)^{2}+\gamma^{2} \theta_{0}\left(K+h_{s} \cos \phi_{0}\right) \\
& +\frac{h_{y} \gamma^{2} u}{C} \sin \phi_{0} \frac{\mathrm{d} \phi_{0}}{\mathrm{~d} s},
\end{aligned}
$$

and for very small external field we can take

$\frac{\mathrm{d}^{2} \phi_{0}}{\mathrm{~d} s^{2}}=\gamma^{2} h_{s} \sin \phi_{0}$.

(21) as

$(L+\lambda) \theta_{0}=b \operatorname{sech}^{2} \chi \tanh \chi$,

where

$L=-\frac{\mathrm{d}^{2}}{\mathrm{~d} \chi^{2}}+\left(1-6 \operatorname{sech}^{2} \chi\right)$.

Now equation (23) is solved by making use of the complete orthonormal eigenfunctions $\psi_{n}(\chi)$ defined by $L \psi_{n}=\varepsilon_{n} \psi_{n}$. One has

$\varepsilon_{0}=-3, \quad \psi_{0}=\sqrt{3} / 2 \operatorname{sech}^{2} \chi$,

$\varepsilon_{1}=0, \quad \psi_{1}=\sqrt{3 / 2} \operatorname{sech} \chi \tanh \chi$,

$\varepsilon_{k}=1+k^{2}$,

$\psi_{k}=\left[\left(1+k^{2}\right)\left(4+k^{2}\right)\right]^{-1 / 2}$ $\times \mathrm{e}^{\mathrm{i} k \chi}\left(1+k^{2}+3 \mathrm{i} k \tanh \chi-3 \tanh ^{2} \chi\right)$.

Expanding $\theta_{0}$ as

$\theta_{0}(\chi)=\alpha_{0} \psi_{0}+\alpha_{1} \psi_{1}+\int \mathrm{d} k \alpha_{k} \psi_{k}$,

and evaluating integrals to obtain the coefficients $\alpha_{n}$ we find

$\theta_{0}(\chi)=\sqrt{\frac{3}{2}} \frac{b \pi}{8 \lambda} \operatorname{sech} \chi \tanh \chi+F(\chi, \lambda)$,

where

$$
F(\chi, \lambda)=-\frac{\mathrm{i} b \pi}{8} \int_{-\infty}^{\infty} \frac{\mathrm{d} q \mathrm{e}^{\mathrm{i} q \chi} q^{2}\left(1+q^{2}+3 \mathrm{i} q \tanh \chi-3 \tanh ^{2} \chi\right) \operatorname{cosech}(\pi q / 2)}{\left(1+\lambda+q^{2}\right)\left(1+q^{2}\right)}
$$

After some lengthy algebra, the expression for the energy of the slowly moving kink takes the form

$$
\begin{aligned}
E_{x y}^{u}= & \gamma J S^{2} \sqrt{h_{s}}\left\{8+\left(1-\frac{u^{2}}{C^{2}}\right)\left(\frac{4}{3}+\frac{17 h_{s}}{15}\right) \lambda\right. \\
& \left.+\frac{\pi^{2} h_{y}^{2}}{120} \frac{u^{2}}{C^{2}} \int_{-\infty}^{\infty} \frac{\mathrm{d} q q^{4} \operatorname{cosech}^{2}(\pi q / 2)\left(24+15 q^{2}+6 q^{4}\right)}{\left(1+\lambda+q^{2}\right)\left(1+q^{2}\right)}\right\} \mathrm{O}\left(\theta_{0}^{2}\right) .
\end{aligned}
$$

Equation (19) has solution

$\phi_{0}(s)=4 \arctan \left[\exp \left( \pm \gamma \sqrt{h_{s}} s\right)\right]$.

Inserting equation (20) into equation (18) we obtain $\frac{\mathrm{d}^{2} \theta_{0}}{\mathrm{~d} \chi^{2}}=\theta_{0}\left(\lambda+1-6 \operatorname{sech}^{2} \chi\right)-b \operatorname{sech}^{2} \chi \tanh \chi$,

where

$\chi=\gamma \sqrt{h_{s}} s, \quad b=\frac{4 h_{y} \gamma u}{\sqrt{h_{s}} C}$

Following Mikeska and Osano [5] we write equation
Now to study the stability of the kink solution (27) we will consider the behaviour of the small oscillations in the presence of a single static kink, i.e.

$\phi(\chi, t)=\phi_{0}(\chi)+q(\chi) \mathrm{e}^{-\mathrm{i} w t}$,

$\theta(\chi, t)=\frac{\pi}{2}+\theta_{0}(\chi)+p(\chi) \mathrm{e}^{-\mathrm{i} w t}$.

Substitution of equations (30) into equations (8) and (9), linearization in $p(\chi), q(\chi)$ and $\theta_{0}(\chi)$ leads to the following eigenvalue equations

$$
\begin{aligned}
\frac{\mathrm{d}^{2} p(\chi)}{\mathrm{d} \chi^{2}}= & \left(1+\lambda-\frac{w^{2}}{h_{s} C^{2}}-6 \operatorname{sech}^{2} \chi\right) p(\chi) \\
& -\frac{2 \mathrm{i} w h_{y}}{h_{s} C} \operatorname{sech} \chi \tanh \chi q(\chi),
\end{aligned}
$$




$$
\begin{aligned}
\frac{\mathrm{d}^{2} q(\chi)}{\mathrm{d} \chi^{2}}= & \left(1-\frac{w^{2}}{h_{s} C^{2}}-2 \operatorname{sech}^{2} \chi\right) q(\chi) \\
& +\frac{2 \mathrm{i} w h_{y}}{h_{s} c} \operatorname{sech} \chi \tanh \chi p(\chi) .
\end{aligned}
$$

For $h_{s} \gg h_{y}$ we can neglect the last terms on the right hand side of equation (31) and (32). Equation (31) becomes

$\frac{\mathrm{d}^{2} p(\chi)}{\mathrm{d} \chi^{2}}-\left(1-6 \operatorname{sech}^{2} \chi\right) p(\chi)=\left(\lambda-\frac{w_{1}^{2}}{h_{s} C^{2}}\right) p(\chi)$.

This equation possesses two bound-states solutions with energies [6]

$w_{11}^{2}=\lambda h_{s} C^{2}$ and $w_{12}^{2}=(\lambda-3) h_{s} C^{2}$,

and continuum states

$p_{k}(\chi)=\frac{\left.1+k^{2}+3 i k \tanh \chi-3 \tanh ^{2} \chi\right) \mathrm{e}^{\mathrm{i} k \chi}}{\left[\left(1+k^{2}\right)\left(4+k^{2}\right)\right]^{1 / 2}}$

with energy

$w_{1 k}^{2}=\left(1+\lambda+k^{2}\right) h_{s} C^{2}$.

As we can see from equation (34), for $\lambda<3$ the static kink becomes unstable to out-of-plane motion. This instability is related to a diverging out-of-plane spin component owing to the motion of the soliton. Of course equations (34) to (36) are exact in the case $h_{y}=0$, when we have an easy-plane antiferromagnet in an external staggered field and its behaviour is similar to what happens in the easy-plane ferromagnetic chain in an external uniform field $[5,7,8]$. For $\lambda<3$ the kink can lower its energy if it moves with an arbitrary small velocity $u$.
For equation (33) we write

$$
\frac{\mathrm{d}^{2} q(\chi)}{\mathrm{d} \chi^{2}}-\left(1-2 \operatorname{sech}^{2} \chi\right) q(\chi)=-\frac{w_{2}^{2}}{h_{s} C^{2}} q(\chi) .
$$

This equation has a bound-state with energy $w_{21}=0$, related to translational invariance, and continuum states

$q_{k}(\chi)=\frac{(k+\mathrm{i} \tanh \chi) \mathrm{e}^{\mathrm{i} k \chi}}{\left(1+k^{2}\right)^{1 / 2}}$,

with energy

$w_{2 k}^{2}=\left(1+k^{2}\right) h_{s} C^{2}$.

We have found that the nonlinear dynamics of the easy-plane antiferromagnetic chain in a staggered field, presents an instability similar to what happens to the easy-plane ferromagnetic chain in an external magnetic field. Possibly more detailed studies would lead to conclusions similar to the ones obtained in [5].

Acknowledgement - This work was partially supported by CNPq, FINEP and CAPES.

\section{REFERENCES}

1. J.P. Boucher, Hyperfine Int. 49, 423 (1989).

2. H.J. Mikeska, J. Phys. C13, 2913 (1980).

3. J.A. Holyst, Z. Phys. B74, 341 (1989).

4. C.A. Condat, R.A. Guyer \& M.D. Miller, Phys. Rev. B27, 474 (1983).

5. H.J. Mikeska \& K. Osano, Z. Phys. B52, 111 (1983).

6. J.F. Curie. J.A. Krumhansl, A.R. Bishop \& S.E. Trullinger, Phys. Rev. B22, 477 (1980).

7. P. Kumar, Phys. Rev. B25, 483 (1982).

8. E. Magyari \& H. Thomas, Phys. Rev. B25, 531 (1982). 